\title{
Calidad del agua y posibles tecnologías de tratamiento en las áreas urbanas de Colón, Panamá: Agua Colón
}

\author{
Kurt PhD, Zohre \\ INDICASAT-AIP \\ Ciudad de Panamá, Panamá \\ zohrekurt@gmail.com \\ Coatney, John Michael \\ Future Scientist \\ Ciudad de Panamá, Panamá \\ johnmichael@futurescientist.org \\ Silvera, Tancy \\ Universidad Tecnológica de Panamá \\ Ciudad de Panamá, Panamá \\ tancysilvera01@gmail.com
}

\section{Abstract}

The Agua Colón Project sought to study the various subterranean water sources in the Santa Isabel district of Costa Arriba, Colón to better understand both the hydrographical distribution of water sources as well as localize the contamination points, if there are any, that affect these potable water sources. The project had three objectives: 1 . Determine the quality of water and pollutants in water wells in rural communities of the District of Santa Isabel; 2. Evaluate biological processes to eliminate pesticide contamination; 3 . Educate the community in general about water quality and how to maintain drinking water through surveys and information sessions on strategies to detect and treat contamination. Through water sampling of the different water sources, both directly from the wells and local river sources as well as from selected residential faucets, the water being consumed by the local populous was analyzed. Water analysis was carried out in a lab provided by Florida State University - Panama Campus. Sampling trips were carried out once a month for the duration of the project. The results found that residents of the coastal communities of Santa Isabel are consuming less than adequate water, and household decontamination methods must be the focus of an educational intervention in these communities. 
Keywords: Water security, community development, education, water contamination, Colón

\section{Resumen}

El proyecto Agua Colón buscaba estudiar las distintas fuentes de agua subterránea en el distrito de Santa Isabel, Costa Arriba, Colón para mejor entender tanto la distribución hidrográfica de las fuentes de agua, así como localizar los distintos puntos de contaminación, si hay, que afecten estas fuentes de agua potable. El proyecto tiene tres objetivos: 1. Determinar la calidad de agua y contaminantes en los pozos de agua en comunidades rurales del distrito de Santa Isabel; 2. Evaluar los procesos biológicos para eliminar contaminación por pesticidas; 3 . Educar a la comunidad en general sobre la calidad de agua y cómo mantener el agua potable a través de encuestas y sesiones informativas sobre estrategias para detectar y tratar la contaminación. A través de el muestreo de distintas fuentes de agua, directamente de los pozos y los ríos, así como grifos seleccionados de viviendas, se analizó el agua consumida por la población local. Los análisis de agua se realizaron en un laboratorio de Florida State University - Campus Panamá. Los viajes de muestreo fueron realizados una vez por mes por la duración del proyecto. Los resultados encontraron que los residentes de las comunidades costeñas de Santa Isabel están consumiendo una calidad de agua menos de adecuada, y que métodos caseros de descontaminación deben ser el enfoque de una intervención educativa en estas comunidades.

Palabras claves: Seguridad hídrica, desarrollo comunitario, educación, contaminación hídrica, Colón.

\section{INTRODUCCIÓN}

La región costeña de Costa Arriba, Colón ha experimentado problemas de seguridad hídrica por años. Estos problemas surgieron no por una falta de fuentes hídricas en la región, sino por una falta de buena administración y mala calidad de agua debido a esta mala administración. Específicamente, la comunidad de Miramar, ubicada por la costa cerca del fin noreste de la provincia panameña de Colón, tiene dos fuentes de las que sacan agua potable. Sin embargo, los residentes tienden a beber agua embotellada y el agua que usan para sus actividades diarias (lavar ropa, bañarse, asear, lavarse las manos) viene del grifo. 
Las preguntas que el proyecto que contestar son: ¿ Por qué los residentes compran su agua bebible cuando muchos tienen agua de servicio $24 / 7$ desde su grifo? ¿Está contaminada el agua potable? Si es así, ¿qué es lo que causa la contaminación? El proyecto Agua Colón ha trabajado en analizar estas fuentes donde la gente saca su agua potable y ha desarrollado una estrategia educativa para alentarle a la población a que descontaminen su agua en casa para reducir los costos de comprar agua embotellada y proveer mayor seguridad hídrica.

\section{MÉTODO}

\section{A. Muestreo de agua}

Cada mes, muestras de las distintas fuentes de agua fueron llevados al laboratorio de Florida State University para los análisis.

Se hacían análisis en el campo también, con un laboratorio portátil para uso en el campo.

\section{B. Coordinación comunitaria}

Organizando con el líder comunitario involucrado con el proyecto, se organizó una reunión comunitaria regional para educarle a la población sobre los resultados del proyecto y cómo afecta la vida de los miembros comunitarios.

Como una intervención educativa, se les enseñó a las personas de las comunidades sobre métodos caseros para filtrar, descontaminar, y almacenar correctamente el agua potable.

\section{RESULTADOS}

\section{A. Figuras}

Fig. 1

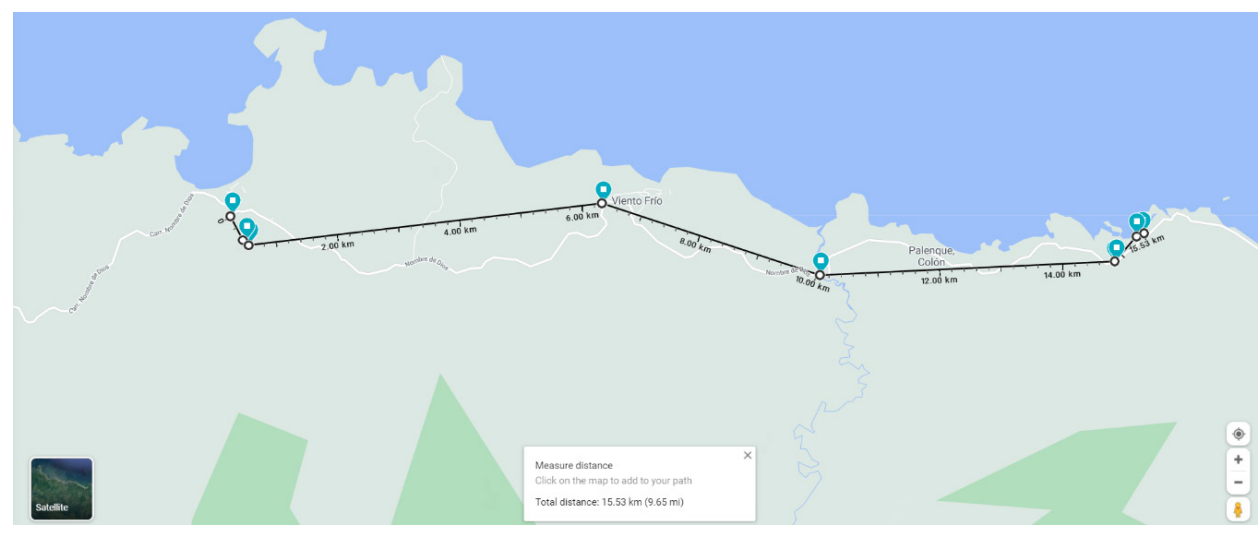

Mapa de fuentes analizadas 


\section{B. Tablas}

Tabla 1. Resultados de análisis 2020-2021

\begin{tabular}{|c|c|c|c|c|c|c|c|c|c|c|c|c|c|c|}
\hline & \multicolumn{4}{|c|}{ Enero } & \multirow{2}{*}{\multicolumn{2}{|c|}{$\begin{array}{r}\text { Noviembre } \\
13 / 11 / 2020 \\
\end{array}$}} & \multirow{2}{*}{\multicolumn{2}{|c|}{$\begin{array}{c}\text { Enero } \\
19 / 01.2021 \\
\end{array}$}} & \multirow{2}{*}{\multicolumn{2}{|c|}{$\begin{array}{c}\text { Febrero } \\
19 / 02 / 2021 \\
\end{array}$}} & \multirow{2}{*}{\multicolumn{2}{|c|}{$\begin{array}{c}\text { Marzo } \\
30 / 03 / 2021\end{array}$}} & \multirow{2}{*}{\multicolumn{2}{|c|}{$\begin{array}{c}\text { Abril } \\
19 / 04 / 2021 \\
\end{array}$}} \\
\hline & \multicolumn{2}{|c|}{$01 / 07 / 2020$} & \multicolumn{2}{|c|}{$29 / 01 / 2020$} & & & & & & & & & & \\
\hline & PMR & RPMR & PMR & RPMR & PMR & RPMR & PMR & RPMR & PMR & RPMR & $P M R$ & RPMR & PMR & RPMR \\
\hline $\mathrm{pH}$ & 7,06 & 7,24 & 7,65 & 7,83 & 6,66 & 6,71 & 6,82 & 6,82 & 6,67 & 7,20 & 6,58 & 6,40 & 7,91 & 8,36 \\
\hline Conductivity (us/cm) & 411 & 161 & 397 & 209 & 284,3 & 48 & 274 & 62 & 117 & 110 & 234 & 168 & 363 & 193,1 \\
\hline Dureza (ppm) & 156,6 & 104,4 & 172 & 103,2 & 120,4 & 154,8 & 172 & 103,2 & 120 & 103 & 137,6 & 103,2 & 120,4 & 85,2 \\
\hline Cloruro (ppm) & 60 & 40 & 60 & 40 & 60 & 40 & 80 & 40 & 40 & 40 & 60 & 40 & 60 & 40 \\
\hline Alcalinidad (Ppm) $\mathrm{CaCO} 3$ & 128 & 114 & 133 & 117 & 217 & 121 & 165 & 90 & 50,3 & 44,1 & 137 & 105 & 147 & 84,6 \\
\hline Turbidez (NTU) & 0,963 & 3,85 & 2,01 & 2,16 & 0,963 & 3,85 & 0,856 & 1,81 & 0,214 & 1,21 & 0,931 & 6,29 & 2,56 & 2,7 \\
\hline Turbidez (JTU) & & & & & & & & & & & & & & \\
\hline$\%$ Salinidad & 0 & 0 & 0 & 0 & 0 & 0 & 0 & 0 & 0 & 0 & 0 & 0 & 0 & 0 \\
\hline Coliformes & positive & positive & negative & positive & positive & positive & positive & positive & positive & positive & positive & positive & positive & positive \\
\hline E. coli & negative & positive & negative & positive & negative & positive & positive & positive & negative & positive & negative & positive & negative & positive \\
\hline Hierro (ppm) & 0,312 & 0,091 & 0,479 & 0,112 & 0,312 & 0,091 & 0,476 & 0,106 & 0,106 & 0,056 & 0,446 & 0,193 & 0,548 & 0,162 \\
\hline Sulfato, rango bajo (ppm) & 9,32 & 2,89 & 7,01 & 2,38 & 9,32 & 2,89 & * & * & 3,74 & 2,18 & 8,54 & 3,02 & 11,6 & 3,03 \\
\hline Nitrito (ppm) & $-0,184$ & $-0,183$ & $-0,181$ & $-0,184$ & $-0,184$ & $-0,183$ & * & * & $-0,174$ & $-0,175$ & $-0,176$ & $-0,169$ & $-0,043$ & $-0,056$ \\
\hline libre de cloro (ppm) & 0,01 & 0,01 & 0,01 & 0,01 & 0,01 & 0,01 & 0 & 0,01 & $-0,02$ & 0 & 0,03 & $-0,09$ & 0,09 & $-0,03$ \\
\hline Nitrato (ppm) & 0,191 & 0,239 & 0,198 & 0,219 & 0,191 & 0,239 & 0,55 & 0,547 & 0,117 & 0,158 & 1,12 & 1,41 & 0,138 & 0,195 \\
\hline Cobre (ppm) & 0,167 & 0,083 & 0,035 & 0,026 & 0,167 & 0,083 & 0,02 & $-0,09$ & $-0,007$ & $-0,017$ & $-0,016$ & 0,003 & 0,026 & 0,01 \\
\hline
\end{tabular}

\section{CONCLUSIONES}

Ya que se han encontrado coliformes en el pozo de Miramar, el agua no se presenta potable para el consumo humano. Hace falta que los administradores del sistema de agua solucionen el problema con una intervención directa de descontaminación al tanque de depósito o que los miembros comunitarios adopten intervenciones caseras de descontaminación y filtración. Al final, el problema de la seguridad hídrica en el distrito de Santa Isabel no tiene que ver con la cantidad de fuentes de agua disponibles para el uso comunitario, sino con la administración de esas fuentes y las intervenciones de tratamiento del agua para que sea potable y consumible.

\section{Referencias}

[1] CONAGUA. (n.d.). Retrieved September 28, 2020, from http://conagua.gob.pa/images/documentos/ Plan/PRIMER-PLAN-NACIONAL-DE-SEGURIDAD-HIDRICA-DE-LA-REPUBLICA-DE-PANAMA.pdf

[2] Gadgil, A. (1998). Drinking water in developing countries. Annual review of energy and the environment, 23(1), 253-286.

[3] Hope, R., \& Rouse, M. (2013). Risks and responses to universal drinking water security. Philosophical Transactions of the Royal Society A: Mathematical, Physical and Engineering Sciences, 371(2002), 20120417.

[4] News, R. (2019). 1 in 3 people globally do not have access to safe drinking water - UNICEF, World Health Organization. Retrieved September 28, 2020, from https://www.who.int/news-room/ detail/18-06-2019-1-in-3-people-globally-do-not-have-access-to-safe-drinking-water-unicef-who

[5] Rahman, M. T. U., Rasheduzzaman, M., Habib, M. A., Ahmed, A., Tareq, S. M., \& Muniruzzaman, 
S. M. (2017). Assessment of fresh water security in coastal Bangladesh: An insight from salinity, community perception and adaptation. Ocean \& Coastal Management, 137, 68-81.

[6] Vörösmarty, C. J., McIntyre, P. B., Gessner, M. O., Dudgeon, D., Prusevich, A., Green, P., ... \& Davies, P. M. (2010). Global threats to human water security and river biodiversity. Nature, 467(7315), 555561.

\section{Autorización y Licencia CC}

Los autores autorizan a APANAC XVIII a publicar el artículo en las actas de la conferencia en Acceso Abierto (Open Access) en diversos formatos digitales (PDF, HTML, EPUB) e integrarlos en diversas plataformas online como repositorios y bases de datos bajo la licencia CC:

Attribution-NonCommercial-ShareAlike 4.0 International (CC BY-NC-SA 4.0) https://creativecommons. org/licenses/by-nc-sa/4.0/.

Ni APANAC XVIII ni los editores son responsables ni del contenido ni de las implicaciones de lo expresado en el artículo. 